Abdelkhalek, Eva S., Elsibai, Mohamad D., Ghosson, Ghida K., and Hamzeh, Farook R. (2019). "Analysis of Visual Management Practices for Construction Safety" In: Proc. 27th Annual Conference of the International. Group for Lean Construction (IGLC), Pasquire C. and Hamzeh F.R. (ed.), Dublin, Ireland, pp. 1069-1080. DOI: https://doi.org/10.24928/2019/0175. Available at: 〈www.iglc.net>.

\title{
ANALYSIS OF VISUAL MANAGEMENT PRACTICES FOR CONSTRUCTION SAFETY
}

\author{
Abdelkhalek, Eva S. ${ }^{1}$, Elsibai, Mohamad D. ${ }^{2}$, Ghosson, Ghida K. ${ }^{3}$, and Hamzeh, \\ Farook R. ${ }^{4}$
}

\begin{abstract}
Lean Construction's main goal is adding value to a project and minimizing waste. Accordingly, incidents that cause injuries or deaths are considered waste. This reveals the interdependent relationship between lean and safety. Here comes the importance of visual management (VM) as a way for communicating information using the right visual tools at the right time for the right situation in all construction projects. It is crucial as it improves efficiency, ensures transparency, establishes better communication and reduces safety risks. This paper highlights the importance of VM in the workplace for construction crews as well as its relation to safety. In this study, case studies and surveys conducted in Lebanon on building construction projects are presented, analyzed and discussed to show the need for VM by workers and engineers to avoid hazardous situations. Results indicate the importance of VM in maintaining safety, illustrate that poor visualization causes many accidents, and show that construction safety in Lebanon can be improved by enhancing VM practices.
\end{abstract}

\section{KEYWORDS}

Safety, Lean Construction, Visual Management, Visual Tools, Visual Orders.

\section{INTRODUCTION}

VM is a way for communicating information using different visual tools for collaborative and integrated planning and control. Liff (2007) stresses on the importance of VM. As described by him, an open organization that encourages sharing information, celebrates achievements as well as failures, supports the employees and strives for a better performance is an organization that uses VM. VM had been attracting research interest for many years. It is one of the most important approaches, simplest and easiest way to share

1 Master Candidate, Civil and Environmental Engineering Dept., American University of Beirut, +96170157034, esa17@mail.aub.edu

2 Master Candidate, Civil and Environmental Engineering Dept., American University of Beirut, +96171152853, mde04@mail.aub.edu

${ }^{3}$ Master Candidate, Civil and Environmental Engineering Dept., American University of Beirut, +96170657040,gkg01@ mail.aub.edu

${ }^{4}$ Associate Professor, Civil and Environmental Engineering Dept., University of Alberta, Canada, hamzeh@ualberta.ca 
and communicate information ensuring clarity and good understanding (Liker 2004). One of the reasons for using VM is safety management where many papers and studies have investigated its importance (Enshassi et al., 2014, Awada et al., 2016). This paper analyzes VM practices for construction safety in Lebanon. Here, so many questions arise. How effective are safety visuals in a construction project? Is it possible to mitigate the risks being faced in the Lebanese Construction Industry using good VM? To which extent preventing accidents depend on good visualization? Thus, the direct impact of VM on safety will be discussed in terms of affecting conditions, direct reasons for risks and recommendations to implement a good safety program.

Construction sites are usually large and dynamic environments where different crews move around, the layout undergoes several modifications throughout the project, and the construction itself might become a visual barrier (Formoso et al., 2002). The construction industry is known as one of the most dangerous industries that witnesses hazardous and fatal accidents (Awwad, 2015). For this reason, safety should be improved in an attempt to reduce and eliminate safety accidents from occurring (Ahmed et al., 2000). Besides, as indicated by Howell et al. (2002), there is a clear relationship between lean and safety. On one hand, Lean's main principles are eliminating waste and ensuring one piece flow and continuous work. On the other, safety accidents interrupt the flow of work. Reducing these accidents by maintaining an adequate safety program will reduce waste. Many causes can lead to safety accidents. Proper VM plays a great role in ensuring a safe workplace whereas the lack of VM and poor visualization leads to unsafe situations which cause accidents (Shrestha et al., 2011).

The main objective behind proper visualization is to effectively communicate information using visuals. These visuals may be signs, labels, etc. that are basically displayed all over the construction site transferring certain messages and information that are needed to prevent safety accidents (Saurin, et al., 2001). VM, which is exchanging data using visual tools, has a historical background. In Japan, the different approaches and tools for Lean Construction such as VM including visual orders that are known as the 5S (sort, stabilize, shine, standardize and sustain) started developing in the mid-1950s (Fabrizio and Tapping, 2006). However, many companies adopted 6S rather than 5S; $6 \mathrm{~S}$ being the known $5 \mathrm{~S}$ plus an additional $\mathrm{S}$ referring to safety. This is to focus on its importance to avoid hazardous situations in the construction workplace. Moreover, a number of previous studies indicated that there is a clear relationship between applying the $5 \mathrm{~S}$, whether in the workplace or any other, and maintaining safety performance (Saari, 1989).

This paper presents an investigation on the significance of implementing VM in construction projects in Lebanon and how it enhances safety. A part of this paper covers VM tools and their importance regarding safety. Whereas, another part presents different case studies that are briefly described. Moreover, surveys conducted on construction projects show quantitative and qualitative data indicating the extent and effectiveness of using safety visuals in Lebanon. 


\section{BACKGROUND ON VISUAL MANAGEMENT}

Effective communication is important for learning and improving. VM can ensure communication between different parties and employees to achieve lean construction goals. $\mathrm{VM}$ is the managerial strategy (neither a technique nor a tool) of consciously integrating visual (sensory) information into processes to increase process transparency and selfmanagement capabilities at a workplace (Grief, 1991). Rather than relying on the workers' reporting of incidents deviating from safe norms (Aslesen et al., 2014), VM is mainly shaped through the reliance on visual tools and orders which are the first steps on the VM ladder.

\section{VISUAL TOOLS}

As identified by Galsworth (1997), visual tools are divided into four categories which are essential for implementing VM. These four categories are: visual indicators, visual signals, visual controls and visual guarantees. The aforementioned visual tools are crucial to achieve safety management, and as the saying goes, a picture is worth a thousand words. First, visual indicators pass crucial information from management to operational level intending to affect human's behaviour. Examples of visual indicators are charts, photos, value stream maps, etc. Secondly, visual signals are used to provoke a response to the eyesight and interpret the surrounding environment enforcing high human control and directing human's behaviour. They tend to grab attention for some essential points such as traffic light or and on boards. The third category is visual controls that show deviations and help people see how they do their jobs. They enforce full human control by limiting the response to a specific height, width, length, quantity etc. Some examples of visual controls are road lines, maintenance, Kanban, colour coding, display of safety regulations. Last but not least, visual guarantees ensure that everything is done in the right way enabling only the correct outcome. They are known as mistake proof or Poka-yoke striving to eliminate human errors such as machines that stop operating at an identified time or pumps that stop when tanks are filled.

Figure 1 shows the four different types of visual tools used on different sites in Lebanon

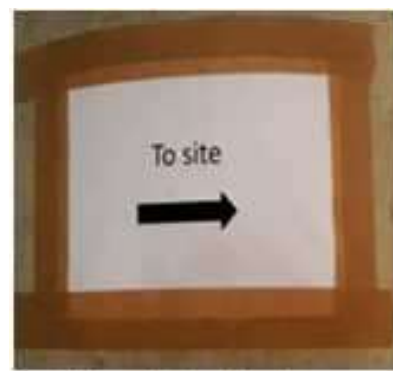

Visual Indicator

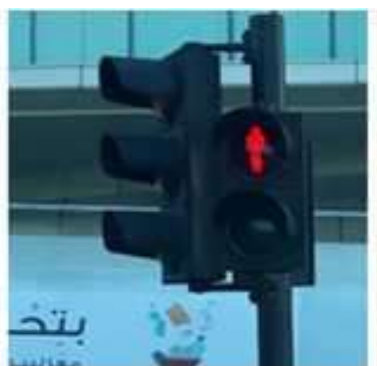

Visual Signal

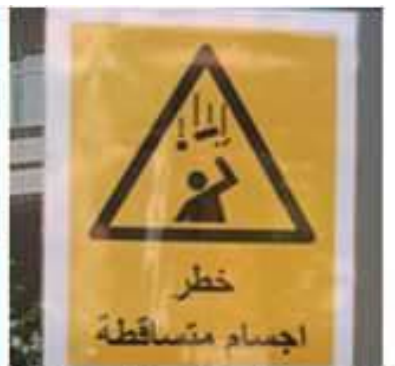

Visual Control Visual Guarantee

Figure 1- The four types of Visual Tools inspired from (Tezel, 2015)

\section{VISUAL ORDER-6S}

When applying VM, 5S is a key essential. It's the first step to a visual workplace. In this paper, as mentioned before, safety is added to attain the $6 \mathrm{~S}$. By taking safety into account 
along with $5 \mathrm{~S}$, the aim is not only to keep the workplace neat and organized but to ensure that it is safe at all times. The $6 \mathrm{~S}$ are classified into: (1) Sort is removing all unnecessary items from the workplace. (2) Stabilize is assigning a place for every item or material to be returned to after each use. (3) Shine is cleaning the workplace before and after the work is done. (4) Standardize is following the same steps for the same process and maintaining the level of organization and readiness in the workplace. (5) Sustain is maintaining a correct procedure to build a culture of continuous improvement. (6) Safety is eliminating all hazards and verifying that suitable control measures are in place (Sukdeo 2007).

Figure 2 shows a framework for VM implementation. It illustrates that by implementing visual orders then going up to implement all layers reaching visual guarantees, a visual workplace is achieved.

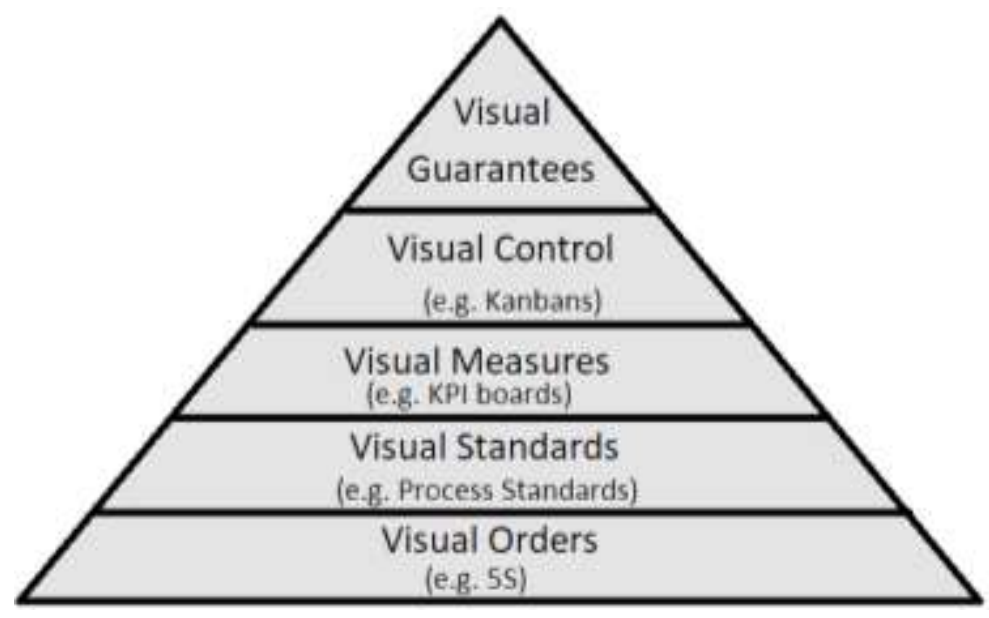

Figure 2- VM Implementation Framework inspired from (Galsworth, 2005)

\section{METHODOLOGY AND METHODS}

The methodology of this paper presents a mixed approach of case studies' analysis performed in Lebanon and results' analysis based on conducted surveys with construction professionals.

First, the case studies' analysis presents 12 building construction sites in Lebanon that differ by type and total built area.

Furthermore, a well-defined procedure for data collection on the mentioned case studies is adopted to analyse the availability and usage of VM and its tools in Lebanon. This procedure consists of the following steps: (1) visiting different projects, (2) meeting multidisciplinary engineers and project managers, (3) interviewing 31 of them and (4) filling the surveys which include questions answered by the interviewed parties. Data collection lasted for over 20 days and the respondents completed the surveys during our meetings to ensure full understanding of the surveys' statements. A 5 point Likert scale was used to grade the statements. The scale ranges from 1 which corresponds to strongly disagree to 5 which is strongly agree. However, the last question was an open-ended one.

In order to develop the questions of the conducted surveys, a literature review was conducted to indicate factors of good VM. These factors are mainly related to visual orders 
and visual tools. (Enshassi et al., 2014). Once ideal VM strategies, tools and practices are well identified and once large number of accidents caused by poor VM on construction sites are detected, surveys' questions were prepared to investigate the conditions of construction safety in Lebanon.

Then, survey results including the mean and standard deviation of all responses are analyzed in the following section in order to get detailed information about safety management in the country, investigate whether or not projects in Lebanon efficiently use visual tools, know how engineers involve their workers in safety, and get an overview on the importance of right design and utilization of visual tools in decreasing the number of accidents and thus decreasing the number of injuries and deaths. The discussion of the results revealed the importance of VM, visual tools and visual orders in construction sites to ensure safety. It also shows the strong relationship between good VM and safety.

Prior to the conclusion, barriers of VM are revealed based on the engineers'/project managers' responses on questions and on specific observations during site visits. Finally, this study investigates the importance of implementing VM in different projects in the Lebanese construction industry and how it affects safety.

\section{CASE STUDIES}

Table 1 presents a brief description of the case studies that were considered in the surveys. Concerning construction case studies, they differ by their size according to the shown total built area in table (1), where some of them are considered as small scale projects, others are considered as medium scale and large scale projects. Also, the mentioned case studies differ by their type whether commercial or residential. Extent of VM usage varies between slight usage where some visual tools are used and no usage where sites lack any VM tool.

Table 1: Construction Sites' Case Studies

\begin{tabular}{clcc}
\hline Case Study & Type & Total Area $\left.\mathbf{( m}^{2}\right)$ & Extent of VM Usage \\
\hline A & Residential & 2940 & No usage \\
B & Residential & 4500 & Slight usage \\
C & Commercial & 22400 & Slight usage \\
D & Residential & 3750 & No usage \\
E & Residential & 3900 & Slight usage \\
F & Commercial & 7000 & No usage \\
G & Residential & 1350 & No usage \\
H & Residential & 1820 & No usage \\
I & Residential & 2040 & No usage \\
J & Residential & 2760 & No usage \\
K & Commercial & 15000 & Slight usage \\
L & Residential & 2880 & No usage \\
\hline
\end{tabular}

\section{RESULTS}

\section{BUILDING CONSTRUCTION SiteS}

Table (2) summarizes the results of the surveys conducted to investigate the current situation of Lebanon regarding safety in construction sites. The mean $\overline{\mathrm{x}}$ and standard 
deviation $o$ are calculated based on the response of the interviewed engineers/project managers whom their answers were given according to Likert scale.

Table 2: Conducted Surveys related to Construction Sites

\begin{tabular}{clcc}
\hline & Statement & Mean $\overline{\mathbf{x}}$ & Standard Deviation $\boldsymbol{\sigma}^{\prime}$ \\
\hline 1 & The construction site is kept clean & 3.92 & 1.44 \\
2 & Every material/tool in its place & 3.17 & 1.27 \\
3 & Safety nets are available & 3.50 & 1.81 \\
4 & PPE are clearly displayed & 2.1 & 1.44 \\
5 & Workers wear hard hats & 2.00 & 1.81 \\
6 & Workers wear hard and closed shoes & 2.33 & 1.67 \\
7 & Workers often face accidents on site & 4.17 & 1.11 \\
8 & First aid tools location is known \& accessible & 2.83 & 1.34 \\
9 & Slab openings and shafts are marked \& closed & 4.50 & 0.90 \\
10 & All exits are clearly marked & 2.75 & 1.29 \\
11 & All walkways are unobstructed & 3.50 & 1.57 \\
12 & Emergency evacuation indicators are available & 2.00 & 1.28 \\
13 & Disposal procedures are visually displayed & 2.50 & 1.00 \\
14 & Visual tools are used to make waste apparent & 2.42 & 1.00 \\
15 & Caution signs to indicate newly casted areas & 1.75 & 1.14 \\
16 & Safety mistakes are regularly reflected & 3.25 & 1.22 \\
17 & Meetings held to increase safety awareness & 1.67 & 1.07 \\
18 & Major barriers of VM implementation & & Open-Ended \\
\hline
\end{tabular}

\section{DISCUSSION OF THE RESULTS}

This section presents an analytical discussion of the conducted surveys and the mentioned case studies.

\section{BUILDing CONSTRUCTION SiTES}

The study results reveal a significant difference between the visited sites. After a thorough investigation, survey analysis, and results' interpretation, a notable variability was detected where few sites use visual tools and follow safety regulations and many others do not use visual tools neglecting safety measures. Based on what have been seen during site visits, large scale projects are found to show more commitment to VM where workers stick to safety measures shown by visual orders on site more than medium and small scale projects where workers show less or even no commitment. Moreover, results indicated that most engineers/project managers are concerned about reducing waste mainly time and cost, increasing projects value and ensuring a safe workplace but they don't focus on VM techniques as statement (14) in table 2 shows. In the aforementioned statement, the mean is equal to 2.42 illustrating the limited usage of visuals that makes waste apparent. 
Results also reveal that many incidents related to safety occurred in those sites that lack VM. Statement (7) in table 2 shows a very high mean (4.17) revealing the high number of these safety accidents on sites. The site engineers responsible for the later projects stated a number of these safety accidents that they have faced. As mentioned by the engineers, these accidents include falling from heights (scaffolding, roofs, etc.), tools and equipment accidents (cranes, trucks, vibrators etc.), falling objects, eye injuries and injuries caused by nails. So, these studies proved that lack of safety is mainly caused by lack of VM. Regarding the sites where VM is slightly used, the interviews with the engineers showed that accidents rarely occur and if they do, they would not be fatal such as injuries caused by nails. Although many sites don't use visual tools, almost all sites care for having a clean and neat workplace as represented by the mean (3.92) in statement (1).

Another crucial point was mentioned by the engineers/project managers where VM is slightly used is that location of first aid tools is well displayed by using different visual signs, for this reason even when simple accidents occur, these accidents are directly mitigated because first aid tools are easily reached and accessed by everyone. However, the mean (2.83) of statement (8) shows that even the simplest things related to safety are not considered in most of the sites in Lebanon. If there's a little knowledge about VM and its importance, by only using few effective, well displayed and organised signs, the location of first aid kits will be known and accessible by everyone. Likewise, statement (12) also shows a low value of the mean (2.00) indicating the lack of emergency evacuation indicators on site. As a result, this absence of visual tools may cause severe accidents to large number of workers in case of fire.

The mean (1.75) of statement (15) is another low value demonstrating the poor usage of visuals such as caution signs for newly casted areas which leads to unexpected damage, excess work, and more money to fix and repair thus causing waste. The interviewed engineers agreed that a clean site prevents some accidents from occurring, such as slips, trips, and nail wounds. They also confirmed that many accidents resulted from wastes and chaos in the workplace (Awada et al., 2016). However, when asking about organising materials/tools and having everything in its place, the response from most of the interviewed parties was that they do care for organising their tools, but this is neither their priority nor the job of the workers. They answered that there is a person who is in charge of organising the workplace, that's why the average answer of this question is neutral as shown in statement (2).

Figures 3, 4 and 5 show pictures taken during different site visits in Lebanon. These pictures demonstrate the difference witnessed between the visited sites. Figures (3) and (4) were taken from the same site. The 1st figure shows the visual indicators are used to display PPE. Workers in this site adhere to them as illustrated in figure (4) where all workers are wearing the safety hard hat and the high visibility vest. In contrast, figure (5) shows workers not taking safety regulations into account as commitment to PPE due to the absence of indicators. It is to be noted here that some companies force workers to wear PPE by imposing certain measures and regulations. Based on this, another key point should be mentioned which is the lack of indicators, signs and labels such as those related to commitment to PPE on construction sites as indicated by most of the respondents. As the table shows in statement (4), the mean is 2.1 which reveals poor visualization. This 
confirms the importance of having visual tools on construction sites to encourage workers to follow safety regulations and thus reduce accidents. Furthermore, both statements (5) and (6) show very low means, 2 and 2.33 respectively, and this is due to the absence of visual indicators and regulations that force, encourage, and remind the workers of the PPE whenever they are on site, whether inspecting or performing the work.
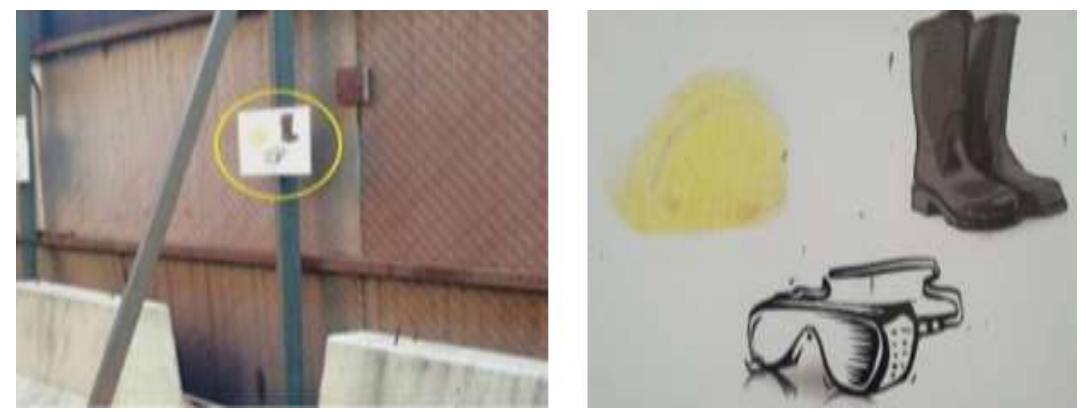

Figure 3- PPE clearly displayed

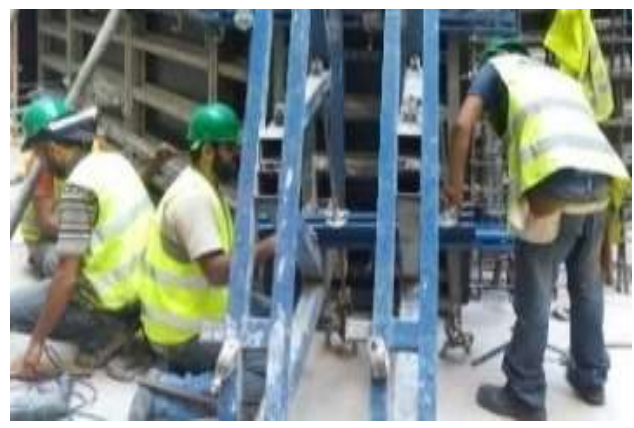

Figure 4-Workers commitment to PPE visual indicators

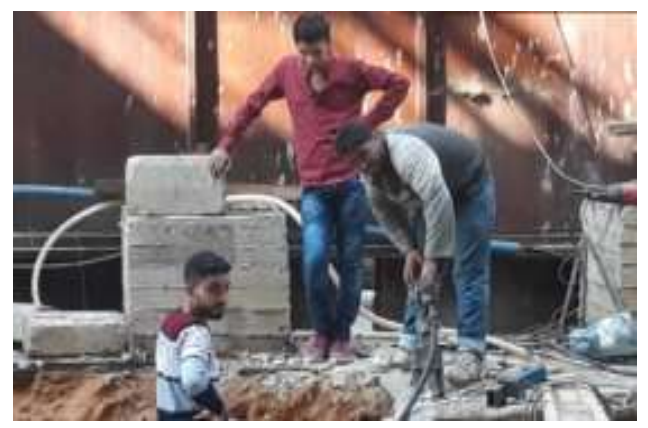

Figure 5- Workers not committed to PPE

Moreover, companies often use visual tools to define the project, project's name, owner, contractor, etc. As displayed in table (2), communication to spread safety awareness between workforces was very limited due to the poor usage of visual tools in the project sites as the mean for statement (17) in the aforementioned table shows. Most of the answers concerning VM on sites reflect the lack of using visual tools, excluding statement (9) that addresses shafts and openings where most of the engineers ensured that shafts and openings should be marked by visual tools and closed by visual barriers. It is the statement with the highest mean (4.5) where it indicates the importance of visual barriers in preventing safety accidents. Also, statements (3) and (11) ended up with an equal mean (3.5) showing that almost all sites use safety nets and keep walkways unobstructed which are major practices on site to avoid fatal accidents such as falling down from high levels or clashing with obstructing objects or materials onto walkways. Confirming to the importance of VM in safety, results align with a previous case study in Lebanon that showed the need for using visual tools to communicating important safety instructions as a key for maintaining a safe environment on construction sites (Awada et al., 2016). 


\section{BARRIERS OF VM ON CONSTRUCTION SITES}

After interviewing more than 12 construction specialists (project managers, site supervisors, etc.) and based on their responses on the open-ended statement which is "major barriers of VM implementation", we can conclude that workers on construction sites have a poor background about VM tools and safety measures. When asked about the major barriers of VM implementation as mentioned in statement (18) in table (2), the interviewed parties mentioned the following barriers: (1) resistance to change, (2) overconfidence, (3) cost, (4) time, (5) lack of governmental regulations, and (6) culture. Traditional contractors, engineers, and workers stick to old methods and resist change, which prevents learning new improvement techniques. Also, those with long experience are overconfident about their work so that they don't obey rules to enhance safety. Besides, they think that it is costly and time consuming to have extra training hours to understand VM tools and orders. Moreover, there are no governmental regulations that encourage workers and managers to implement VM. Last but not least, culture is a major barrier where workers are not educated enough to follow certain safety procedures using VM tools.

\section{CONCLUSION}

This paper investigates the extent of using VM tools on construction building sites in Lebanon. It is clearly shown that VM is an integral part of lean construction, and that better VM leads to better outcomes. So, lean construction emphasizes on using VM to ensure construction safety, thus reducing waste and adding value. Results of the studied cases show that VM needs further improvement in Lebanon and highlight the importance of implementing VM to reduce accidents. After discussing the results, the difference was clear between sites that slightly use visual tools where accidents are limited and not fatal, and sites that never use VM where severe accidents occur frequently.

To conclude, a visual place is an environment that speaks for itself. So, as a recommendation, visuals should be utilized everywhere to direct actions/behaviour and enhance safety for workers, engineers, and the public. In addition, engineers and contractors should dedicate enough time and spend more money on training workers to efficiently use VM tools and reduce accidents. Finally, a lean culture should be cultivated to enhance utilization of VM. Further research is recommended to determine strategies for implementing VM in construction sites so that invested efforts will pay off in more effective results.

\section{REFERENCES}

Ahmed, S., Kwan, J., Ming, F., and Ho, D. (2000), "Site safety management in Hong Kong”, Journal of Management in Engineering, 16(6) 34-42.

Aslesen, S., Sandberg, E., Hamzeh, F.R., and Wehbe, F. (2014) "Improving Safety Performance: Using deviation Reporting as a Source for Continuous Improvement", Proceedings for the 22nd Annual Conference of the International Group for Lean Construction, Oslo, Norway, pp. 1435-1446

Awada, M.A, Lakkis, B.S., Doughan, A.R., and Hamzeh, F. R. (2016). "Influence of Lean Concepts on Safety in the Lebanese Construction Industry." In: Proc. 24th Annual 
Conference of the International Group for Lean Construction, Boston, MA, USA, sect.11 pp. 63-72.

Awwad, R., El Souki, O., and Jabbour, M. (2016). "Construction Safety Practices \& Challenges in a Middle Eastern Developing Country." Safety Science, 83, 1-11.

Brady, D. A. (2014). "Using Visual Management to Improve Transparency in Planning and Control in Construction." PhD Diss., Univ. of Salford, Salford, UK.

Brandalise, F.M.P., Valente, C.P., Viana, D.D., and Formoso, C.T. (2018). "Understanding the effectiveness of Visual Management best practices in construction sites" In: Proc. 26th Annual Conference of the International Group for Lean Construction (IGLC), González, V.A. (ed.), Chennai, India, pp. 754- 763.

Damaj, O., Fakhreddine, M., Lahoud, M., Hamzeh, F. (2016). "Implementing Ergonomics in Construction to Improve Work Performance." In: Proc. 24th Annual Conference of the International Group for Lean Construction, Boston, MA, USA, sect.11 pp. 53-62.

Enshassi, A. \& Abu Zaiter, M. (2014), "Implementation of Lean Tools on Safety in Construction Projects in Palestine" In:,Kalsaas, B.T., Koskela, L. \&Saurin, T.A., 22nd Annual Conference of the International Group for Lean Construction. Oslo, Norway, 25-27 Jun 2014. pp 1205-1218.

Fabrizio, T., and Tapping D. (2006).5S for the Office: Organizing the Workplace to Eliminate Waste.

Formoso, C.T., (M.ASCE), L.S., Cesare, C.D. and Isatto, E.L. (2002). "Material Waste in Building Industry: Main Causes and Prevention," J. Constr. Eng. Manage., ASCE, Vol. 128 No. 4, pp. 316-325.

Galsworth, G. D. (1997). Visual Systems: Harnessing the Power of a VisualWorkplace.New York.

Galsworth, G. D. (2005). Visual Workplace: Visual Thinking. Visual-Lean Enterprise Press, Portland, USA.

Ghossaini, B.M., Dehaini, K.Y., Alruzz, M.A., FakhrEddine, N.A., and Hamzeh, F., (2017). "An Application for Improved Lean Construction Practices" In: Proc. 26th Annual Conference of the International Group for Lean Construction (IGLC), González, V.A. (ed.), Chennai, India, pp. 1195-1205.

Greif, M. (1991). The Visual Factory: Building Participation through Shared Information, Productivity Press, Portland, USA.

Howell, G.A., Ballard, G., Abdelhamid, T.S. \& Mitropoulos, P. (2002)."Working Near the Edge: A New Approach to Construction Safety” In: Formoso, C.T. \& Ballard, G., 10th Annual Conference of the International Group for Lean Construction. Gramado, Brazil, 6-8 Aug 2002. pp 49-60.

Koskela, L., Tezel, A., and Tzortzopoulos, P. (2018). "Why visual management?” In: Proc. 26th Annual Conference of the International. Group for Lean Construction (IGLC), González, V.A. (ed.), Chennai, India, pp. 250-260.

Liff, S. (2007). Seeing Is Believing: How the New Art of Visual Management Can Boost Performance throughout Your Organization.

Liker, J. (2004). The Toyota way: 14 management principles from the world's greatest manufacturer. McGraw-Hill, NY. 
Sukedeo, N. (2017). "The Application of 6S Methodology as a Lean Improvement Tool in an Ink Manufacturing Company". IEEE-IEEM, Singapore.

Saari, J. (1989). "The Effect of Positive Feedback on Industrial Housekeeping and accidents; a Long-term Study at a Shipyard”. International Journal of Ergonomics, 4, 201-211

Shrestha, P. P. Yafantis, E. and Shrestha, K., (2011) “Construction safety visualization”, Proceedings of International Multi-Conference on Engineering and Technological Innovation, ISBN-13: 978-1-936338-36-8, Volume 1, pp. 243-248, IMETI, Orlando, Florida, July 19-21, 2011.

Saurin, T.A., Formoso, C.T. \& Guimaraes, L.B.D.M. (2001) "Integrating Safety into Production Planning and Control Process: An Exploratory Study" In: Ballard, G. \& Chua, D., 9th Annual Conference of the International Group for Lean Construction. Singapore.

Tezel, A., Aziz, Z., Koskela, L., and Tzortzopoulos, P. (2016). "Visual Management Condition in Highways Construction Projects in England" In: Proc. 24th Annual Conference of the International Group for Lean Construction, Boston, MA, USA, sect.6 pp. 133-142.

Tezel, A., Koskela, L., Tzortzopoulos, P., Formoso, C. T., and Alves, T., (2015). "Visual management in Brazilian construction companies: taxonomy and guidelines for implementation." ASCE, J. Mang. Eng, 31(6), 05015001.

Tezel, A., and Aziz, Z., (2015). Visual Controls at the Workface of Road Construction and Maintenance.

Viana, D. D., Bulhoes, I., and Formoso, C. T. (2013). "GuidelinesforIntegrated Planning and Control OF Engineer-to-Order.Proceedings of the 21st Annual Meeting of the International Group for LeanConstruction, Fortaleza, 549-558. 
Abdelkhalek, Eva S., Elsibai, Mohamad D., Ghosson, Ghida K., and Hamzeh, Farook R

1080

Proceedings IGLC-27, July 2019, Dublin, Ireland 